\title{
Applicazione dei processi elettrochimici ai bioreattori a membrane vive incapsulate
}

\author{
Andrea Pierro ${ }^{1}$ \\ ${ }^{1}$ Affiliation not available
}

\begin{abstract}
I bioreattori a membrana (MBR) uniscono nella stessa unità sia il processo biologico che la filtrazione su membrana in sostituzione dei sedimentatori secondari. Gli MBR presentano vari vantaggi rispetto all'impianto convenzionale a fanghi attivi tra cui minor ingombro, migliore qualità dell'effluente e minor produzioni di fanghi. Non mancano, però, degli svantaggi: il primo è rappresentato dai costi elevati dovuti ai consumi energetici e al costo delle membrane, il secondo, ma non meno importante è legato alla formazione del fouling, ossia dello sporcamento delle stesse, che ne riduce il flusso, limitando così l'efficienza del processo. Negli anni sono state studiate diverse tecnologie per porre rimedio a questi svantaggi. L'introduzione delle membrane dinamiche auto-formanti (SFDM) ha permesso di contrastare il problema del costo delle membrane, poiché il loro funzionamento è basato sulla deposizione delle particelle di fango su un modulo di supporto con porosità elevata e realizzato con materiali economici. Lo svantaggio principale di questa tecnologia risiede nella necessità di un tempo iniziale per la completa formazione della membrana dinamica, il quale varia a seconda delle caratteristiche del refluo o delle condizioni operative in cui avviene il processo. Altra soluzione proposta dalla letteratura scientifica per contrastare il problema dello sporcamento della membrana è quella di accoppiare i processi elettrochimici con gli MBR, tale soluzione non solo riduce il fouling di membrana ma aumenta le performance depurative del sistema.
\end{abstract}




\section{Introduzione}

Con il passare degli anni c’è stato un continuo sviluppo nell' ambito del trattamento di depurazione delle acque reflue, il trattamento convenzionale è rappresentato dal processo a fanghi attivi, ma con i vari aggiornamenti e modifiche normative si sono fatte largo nuove tecnologie.

I bioreattori a membrane (MBR) rappresentano un'alternativa all'impianto a fanghi attivi. Questa si basa sull'utilizzo contemporaneo di processo biologico e processo fisico di filtrazione attraverso membrane, con la conseguente scomparsa del sedimentatore all'interno dell'impianto $\left({ }^{1}\right)$. Il sistema MBR comporta dei vantaggi rispetto ai processi a fanghi attivi, tra questi si riportano ${ }^{1}$ :

- Migliore qualità dell'effluente;

- Minori volumi;

- Minor produzione di fanghi;

- Può essere introdotto per adeguare un impianto già esistente.

Oltre, i vantaggi, il processo MBR presenta degli svantaggi, tra cui ${ }^{1}$ :

- Necessità di un pre-trattamento;

- Costi energetici elevati;

- Costi delle membrane;

L'impianto MBR necessita di un pre-trattamento, per eliminare i solidi di maggiore dimensione per evitare che questi vadano subito ad intasare la superficie della membrana ${ }^{2}$. I costi energetici elevati sono legati al controllo del fouling. Il fouling (incrostazione) della membrana è dovuto alla deposizione delle particelle di fango sulla superficie e all'intasamento dei pori $\left({ }^{3} ;{ }^{4}\right)$. Il costo delle membrane, nonostante si sia ridotto negli anni grazie alla loro diffusione, rappresenta un'importante aliquota dei costi di impianto ${ }^{5}$. L'utilizzo di membrane dinamiche auto-formanti rappresenta una tecnologia alternativa al sistema MBR convenzionale in quanto in grado di elimi- 
nare i principali svantaggi che tale tecnologia presenta ${ }^{6}$. La tecnologia delle membrane dinamiche auto-formanti (SFDM) è basata sull'utilizzo di materiale di supporto a basso costo con porosità elevata che va dai 20 ai $100 \mu \mathrm{m}^{7}$. I materiali utilizzati in letteratura sono tessuto non tessuto, tessuto, rete in acciaio inossidabile, rete di nylon, rete di dacron, tessuto filtrante e tubo di ceramica 8. La membrana che si va a formare al di sopra del materiale di supporto si può suddividere in due strati: uno strato superiore di torta e uno inferiore di gel.

La qualità dell'effluente varia al variare delle fasi di formazione, nella fase iniziale essa risulta scarsa, il filtrato si mostra molto denso tale che dovrebbe essere ricircolato nel reattore per essere scaricato ${ }^{9}$. Una volta che la membrana si è formata viene raggiunta un'ottima qualità con concentrazione di SS vicino allo zero, successivamente a causa dell'intasamento del modulo SFDM, lo spessore aumenta e il flusso tende a ridursi, perciò bisogna interrompere il funzionamento e procedere alla pulizia ${ }^{9}$.

Di recente l'applicazione dei processi elettrochimici negli MBR ha rappresentato un'ottima tecnica per il controllo del fouling, essi vengono attuati grazie all'introduzione di due elettrodi perforati immersi nel refluo ${ }^{10}$.

L'applicazione di una corrente provoca dei cambiamenti fisico-chimici nel refluo, con il trasporto di particelle mediante elettrolisi, elettro-migrazione, elettro-osmosi ed elettro-foresi ${ }^{11} ;{ }^{12} ;{ }^{13}$ ). L'elettrolisi dell'acqua produce ioni idrogeno nella parte dell'anodo e ha inizio il meccanismo di elettro-migrazione, ovvero il trasporto di ioni dovuto alla presenza del campo elettrico ${ }^{14}$. Il movimento delle particelle cariche verso l'elettrodo con carica opposta è detto elettro-foresi ${ }^{14}$. L'elettro-foresi e l'elettro-osmosi sono i fenomeni fondamentali nell'elettrocinetica ${ }^{14}$. L'elettroosmosi consiste nel flusso di un liquido ionico per effetto del campo elettrico ${ }^{14}$, questo meccanismo provoca la rimozione dell'acqua legata all'interno dei fiocchi ${ }^{14}$, la quale riduce la resistenza alla filtrazione ${ }^{15}$. Un altro dei processi principali è l'elettrocoagulazione che provoca la formazione di fiocchi più grandi ottenuti dall'assorbimento degli inquinanti i quali riescono ad essere tratte- 
nuti dalla membrana ${ }^{15}$, esso è stato utilizzato anche per il trattamento dei contaminanti emergenti (EC) ${ }^{161718}$.

\section{Setup sperimentale}

Il processo di trattamento e-ESFDMBR utilizzato è il risultato della combinazione della tecnologia SFDM , eMBR e di una nuova tipologia di membrane dinamiche incapsulate. Mentre nella maggior parte degli studi in letteratura si realizza un impianto in scala di laboratorio, utilizzando un refluo sintetico prodotto in loco, nell' attività svolta si è usufruito di un refluo reale in un impianto a scala pilota. L'impianto in scala pilota comprende un reattore con un volume di $100 \mathrm{~L}$. All'interno del reattore biologico sono stati inseriti inoltre due elettrodi collegati a un generatore di corrente per l'applicazione dei processi elettrochimici. Nel reattore è inserito il materiale di supporto per la formazione della membrana, il quale deve essere precedentemente assemblato. Partendo dal telaio centrale in plexiglass, si applica una rete plastica poi un foglio di dacron e successivamente si ripetono nuovamente questi due passaggi per poi chiudere la struttura con il telaio esterno, lo stesso procedimento vale anche per l'altro lato della struttura. Per garantire il fabbisogno di ossigeno per l'attività batterica, ma soprattutto per evitare la rapida formazione di fouling e il deposito dei solidi in fondo al reattore è stato previsto un sistema di aerazione, con un disco in pietra porosa posto sul fondo al di sotto della membrana e dei diffusori a bolle fini distribuiti in maniera radiale all'interno del reattore alimentati da 5 pompe di aerazione. Gli elettrodi hanno entrambi altezza pari a $75 \mathrm{~cm}$, con una corrispondente bagnata pari a $53 \mathrm{~cm}$ e spessore di $2 \mathrm{~mm}$, mentre a variare sono materiale e raggio. L'anodo è in alluminio e il raggio del cilindro è di $20,5 \mathrm{~cm}$, mentre il catodo è in acciaio inossidabile con un raggio di $14,3 \mathrm{~cm}$. Per garantire la circolazione dei liquidi sono state usate 3 pompe dosatrici a membrana, l'attivazione delle pompe di controlavaggio ed effluente avviene tramite uno dei temporizzatori, 1 minuto per la pulizia della membrana e 9 minuti per il prelievo dell'effluente, mentre quella dell'influente era regolata da un galleggiante 
posto all'interno del reattore. L'altro temporizzatore regola l'accensione e lo spegnimento del generatore del campo elettrico con un'intensità di corrente pari a $0,9 \mathrm{~mA} / \mathrm{cm}^{2}(5 \mathrm{~min} \mathrm{ON}$ e $20 \mathrm{~min}$ OFF). Durante l'attività di tirocinio è stato svolto un monitoraggio giornaliero sul trattamento eseguito nell'impianto pilota. L'attività di monitoraggio ha compreso il controllo del livello del refluo all'interno del reattore, la misurazione dei parametri fisici ( $\mathrm{pH}$, temperatura, ossigeno disciolto) e dell'andamento della TMP. Per il corretto svolgimento del processo il livello del liquame doveva mantenersi costante nel tempo. Le analisi in laboratorio sono state realizzate due volte a settimana sui campioni prelevati all'impianto e sono state le seguenti:

- Torbidità;

- Solidi sospesi totali e volatili (SST e SSV);

- Azoto ammoniacale;

- $\mathrm{UV}_{254}$;

- COD;

- Ortofosfati.

\section{Risultati}

Dai dati ottenuti durante il monitoraggio dell'impianto sull' effluente si registra sin dal primo giorno di attivazione una torbidità inferiore alle 5 NTU, per poi scendere al di sotto dell'unità dopo 5 giorni.

Solitamente nei bioreattori a membrana la pressione di transmembrana (TMP) tende a variare nel tempo, con valori bassi all'inizio per poi aumentare a causa del fouling che va a ricoprire la membrana e ostacola la filtrazione. Nel presente studio ciò non avviene, nonostante si sia utilizzata soltanto pulizia fisica mediante controlavaggio, non ricorrendo mai a quella chimica. Questi ri- 
sultati sono dovuti alla combinazione dei processi elettrochimici e della tecnologia SFDM, i primi provocano la riduzione della formazione di fouling, quindi ritardano la necessità di una pulizia chimica, ma ciò che effettivamente la evita è il principio di formazione della membrana SFDM. Essa è formata da due strati, quello superiore è costituito da particelle grandi, mentre lo strato inferiore è uno strato di gel che favorisce la formazione della membrana in seguito alla pulizia fisica ${ }^{9}$. Questi strati fanno da scudo per il materiale di supporto, il quale non viene intasato da piccole particelle che scaturiscono l'esigenza di una pulizia chimica ${ }^{9}$.

Il contenuto di solidi è uno dei parametri più importanti per la valutazione delle prestazioni di un trattamento sulle acque reflue. In particolare sono stati analizzati i solidi sospesi totali (SST) ed i solidi sospesi volatili (SSV). Il reattore ha mostrato un aumento di concentrazione dei solidi dovuto alla degradazione nel tempo dell'anodo . L'applicazione del campo elettrico provoca delle reazioni di ossidazione all'anodo con una liberazione nel refluo di ioni alluminio, i quali vanno a reagire con le particelle di fosfato presenti, facilitandone la rimozione mediante la filtrazione a membrane.

I contaminanti studiati nel presente elaborato sono COD, azoto ammoniacale $\left(\mathrm{NH}_{4}^{+}\right)$e fosfati $\left(\mathrm{PO}_{4}{ }^{3-}\right)$ ed $\mathrm{UV}_{254}$. L'applicazione del campo elettrico ed i conseguenti processi elettrochimici che si sono generati (elettro-coagulazione, elettro-foresi, elettro-osmosi) hanno avuto un'influenza positiva sull'efficienza di rimozione dei contaminanti. Alla fine della sperimentazione i valori medi raggiunti sono stati di $98,03 \%$ per il COD, $99,45 \%$ per $\mathrm{NH}_{4}{ }^{+}, 100 \%$ per $\mathrm{PO}_{4}{ }^{3-}$ e $73,78 \%$ per $\mathrm{UV}_{254}$. 


\section{Conclusioni}

Il trattamento oggetto di studio ha mostrato prestazioni elevate con efficienze di rimozioni superiori ai precedenti trattamenti presenti in letteratura. La combinazione delle varie tecnologie usate all'interno dello stesso reattore si è dimostrata essere una possibile innovazione da attuare nei bioreattori a membrana. L'utilizzo della membrana dinamica auto-formante incapsulata ha permesso di ridurre il costo da fronteggiare rispetto a quella convenzionale e di rallentare la formazione del fouling, dall'altro lato l'applicazione del campo elettrico intermittente ha dato vita a processi elettrochimici che con l'alternanza di condizioni aerobiche e anossiche hanno favorito la rimozione dei contaminanti.

\section{References}

1.Pirozzi, F. Principi di funzionamento e configurazioni degli MBR. in Bioreattori a membrane per il trattamento delle acque reflue (V. Naddeo, V. Belgiorno, 2012).

2.D. Scannapieco, V. B. Utilizzo degli MBR negli interventi di upgrade. in Bioreattori a membrane per il trattamento delle acque reflue (V. Naddeo, V. Belgiorno, 2012).

3.Metcalf, E. Wastewater Engineering: Treatment and Reuse. (McGraw-Hill Companies, 2003).

4.Borea, L. et al.. Wastewater treatment by membrane ultrafiltration enhanced with ultrasound: Effect of membrane flux and ultrasonic frequency. Ultrasonics 83, 42-47 (2018).

5.Krzeminski, P., Leverette, L., Malamis, S. \& Katsou, E. Membrane bioreactors - A review on 
recent developments in energy reduction fouling control, novel configurations, LCA and market prospects. Journal of Membrane Science 527, 207-227 (2017).

6.Millanar-Marfa, J. M. J. et al.. Self-forming Dynamic Membranes for Wastewater Treatment. Separation \& Purification Reviews 1-17 (2021) doi:10.1080/15422119.2021.1887223.

7.Mahat, S. B., Omar, R., Idris, A., Kamal, S. M. M. \& Idris, A. I. M. Dynamic membrane applications in anaerobic and aerobic digestion for industrial wastewater: A mini review. Food and Bioproducts Processing 112, 150-168 (2018).

8.Zhang, Y., Zhao, Y., Chu, H., Dong, B. \& Zhou, X. Characteristics of dynamic membrane filtration: structure operation mechanisms, and cost analysis. Chinese Science Bulletin 59, 247-260 (2013).

9.Fan, B. \& Huang, X. Characteristics of a Self-Forming Dynamic Membrane Coupled with a Bioreactor for Municipal Wastewater Treatment. Environmental Science \& Technology 36, 5245-5251 (2002).

10.Mutamim, N. S. A., Noor, Z. Z., Hassan, M. A. A. \& Olsson, G. Application of membrane bioreactor technology in treating high strength industrial wastewater: a performance review. $D e$ salination 305, 1-11 (2012).

11.Acar, Y. B. \& Alshawabkeh, A. N. Principles of electrokinetic remediation. Environmental Science \& Technology 27, 2638-2647 (1993).

12.Acar, Y. B. et al.. Electrokinetic remediation: Basics and technology status. Journal of Hazardous Materials 40, 117-137 (1995).

13.S. Pamukcu, J. K. W. Electrokinetically enhanced in situ soil decontamination. in Remediation of hazardous waste contaminated soils (D.L. Wise, D.J. Trantolo, 1994).

14.Kim, S.-O., Moon, S.-H., Kim, K.-W. \& Yun, S.-T. Pilot scale study on the ex situ electrokinetic 
removal of heavy metals from municipal wastewater sludges. Water Research 36, 4765-4774 (2002).

15.Ibeid, S., Elektorowicz, M. \& Oleszkiewicz, J. A. Novel electrokinetic approach reduces membrane fouling. Water Research 47, 6358-6366 (2013).

16.Ensano, B. et al.. Removal of Pharmaceuticals from Wastewater by Intermittent Electrocoagulation. Water 9, 85 (2017).

17.Ensano, B. M. B. et al.. Applicability of the electrocoagulation process in treating real municipal wastewater containing pharmaceutical active compounds. Journal of Hazardous Materials 361, 367-373 (2019).

18.Naddeo, V., Landi, M., Scannapieco, D. \& Belgiorno, V. Sonochemical degradation of twenty-three emerging contaminants in urban wastewater. Desalination and Water Treatment 51, 6601-6608 (2013). 
Figure Captions

Figure 1. Impianto in scala pilota 
Figures

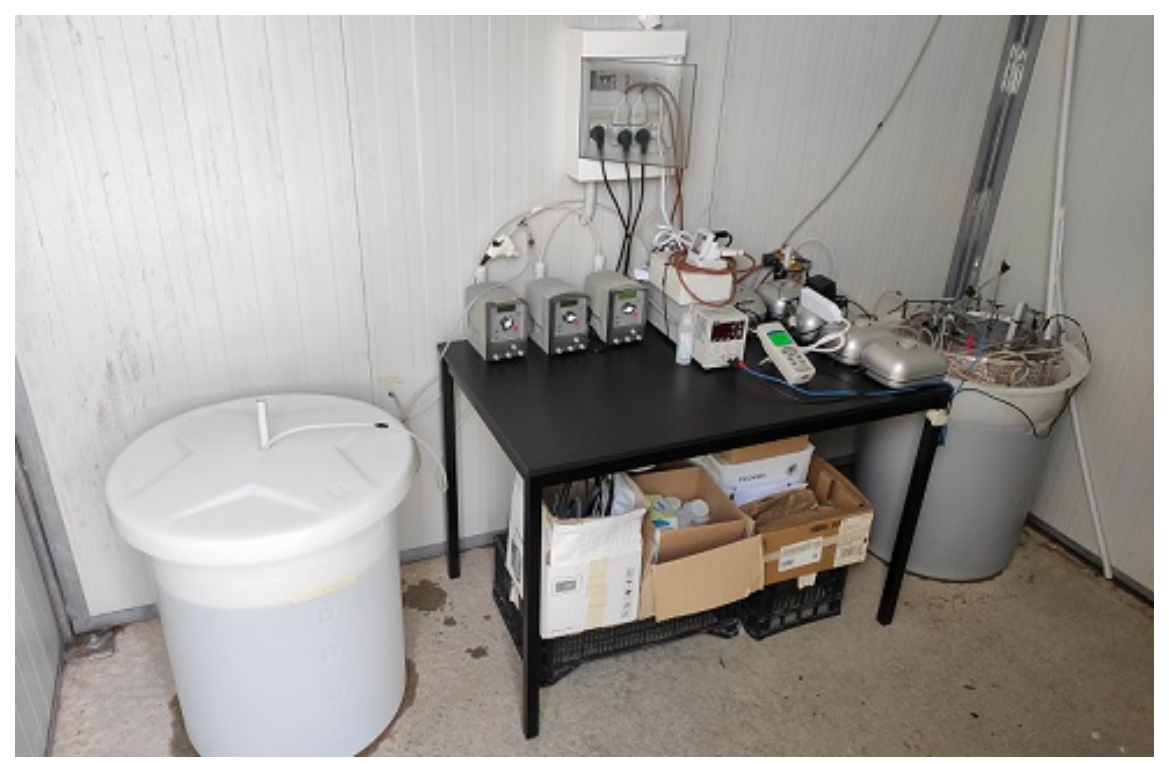

Figure 1: Impianto in scala pilota 